\title{
A produção periódica científica afiliada ao Instituto Nacional da Mata Atlântica (INMA) na base Scopus (2009-2018)
}

\section{The scientific periodical production affiliated to the National Institute of the Atlantic Forest (INMA) at Scopus database (2009-2018)}

\author{
Juliana Lazzarotto Freitas ${ }^{1}$, Fábio Sampaio Rosas ${ }^{2}$, Sérgio Lucena Mendes ${ }^{3}$ \\ ${ }^{1}$ Instituto Nacional da Mata Atlântica (INMA), Santa Teresa, Espírito Santo, Brasil. ORCID: http://orcid.org/0000-0003-2572-9407 \\ 2 Universidade Estadual Paulista (UNESP), Dracena, São Paulo, Brasil. ORCID: https://orcid.org/000-0002-7044-1683 \\ ${ }^{3}$ Instituto Nacional da Mata Atlântica (INMA), Santa Teresa, Espírito Santo, Brasil; Universidade Federal do Espírito Santo (UFES), Vitória, Espírito Santo, \\ Brasil. ORCID: $h$ ttps://orcid.org/0000-0002-7287-6058
}

Autor para correspondência/Mail to: Juliana Lazzarotto Freitas, julilazzarotto@gmail.com

Recebido/Submitted: 18 de julho de 2020; Aceito/Approved: 21 de agosto de 2020

Copyright (C) 2020 Freitas, Rosas \& Mendes. Todo o conteúdo da Revista (incluindo-se instruções, política editorial e modelos) está sob uma licença Creative Commons Atribuição-NãoComercial-Compartilhalgual 3.0 Não Adaptada. Ao serem publicados por esta Revista, os artigos são de livre uso em ambientes educacionais, de pesquisa e não comerciais, com atribuição de autoria obrigatória. Mais informações em http://revistas.ufpr.br/atoz/about/submissions\#copyrightNotice.

\begin{abstract}
Resumo
Introdução: apresenta-se, como objeto de análise, a produção de artigos científicos afiliados ao Instituto Nacional da Mata Atlântica (INMA), nova denominação do Museu de Biologia Professor Mello Leitão (MBML). Objetiva-se reconhecer a visibilidade, o impacto e as relações temáticas e colaborativas estabelecidas pela pesquisa do INMA ao longo de dez anos (2009-2018). Parte-se da seguinte questão: como se configura essa produção científica no que tange a áreas temáticas prioritárias, periódicos, autores, instituições e países mais produtivos e, ainda, qual seu impacto pelas citações recebidas? Metodologia: adotam-se os estudos métricos da informação como metodologia de coleta e análise, identificando-se um corpus de 73 artigos vinculados ao INMA pela afiliação institucional de seus pesquisadores no período de dez anos. Utiliza-se a base de dados Scopus para identificação dessa produção e, também, a base de dados Scival como instrumento para geração de indicadores. Resultados: destaca-se que a produção afiliada ao INMA apresenta índices de internacionalização expressivos, visto que está publicada em $72 \%$ de periódicos internacionais. Além disso, o corpus recebe uma média de citações elevada (4,22 citações por artigo). As pesquisas com maior número de citações apresentam impacto superior à média de citação dos periódicos em que estão veiculadas. As relações interinstitucionais e entre autores constroem-se alinhadas ao escopo temático e histórico do Instituto, consolidando áreas e tendências de pesquisa nos domínios da zoologia e da botânica Conclusão: o perfil da produção científica retratada contribui como elemento estratégico para a consolidação da memória de pesquisa do INMA.
\end{abstract}

Palavras-chave: Instituto Nacional da Mata Atlântica; Análise da produção científica; Estudos Métricos da Informação; Visibilidade científica; Impacto científico; Colaboração científica.

\begin{abstract}
Introduction: this study considers as object of analysis the scientific articles production affiliated to the National Institute of the Atlantic Forest (INMA). It aims to recognize visibility, impact, thematic and collaborative relationships established by researchers afilliated to the Institute over ten years (2009-2018). It starts from the scientific question: how is this scientific production configured regarding priority thematic areas, journals, authors, institutions and most productive countries, and what is its impact on the citations received? Method: it adopts the metric studies of information as methodology, identifying a corpus of 73 articles linked to INMA by the institutional affiliation of its researchers over a period of ten years. It uses the Scopus database to identify this production and also the Scival database as an instrument to generate indicators. Results: it points out that the production affiliated to INMA has high internationalization rates, as it is published in $72 \%$ of international journals. In addition, the corpus receives a high average of citations (4.22 citations per article). The articles with the highest number of citations have greater impact than the average citations of the journals in which they are published. Interinstitutional relations and between authors are built in line with the thematic and historical scope of the institute, consolidating areas and research trends on zoology and botany. Conclusion: the profile of the scientific production portrayed contributes as a strategic element for the consolidation of INMA's research memory.
\end{abstract}

Keywords: National Institute of the Atlantic Forest; Scientific production analysis; Metric Studies of information; Scientific Visibility; Scientific Impact; Scientific Collaboration.

\section{INTRODUÇÃO}

Apresenta-se como objeto de estudo a produção de artigos científicos de pesquisadores afiliados ao Instituto Nacional da Mata Atlântica (INMA), antigo Museu de Biologia Professor Mello Leitão (MBML). O Museu foi fundado em 1949 por Augusto Ruschi, na cidade de Santa Teresa- ES, Brasil, passando da tutela privada à administração pública em 1984 - processo este que culminou na incorporação de seu patrimônio pelo INMA, criado em 2014 e formalmente institucionalizado em 2017, como unidade de pesquisa vinculada ao Ministério da Ciência, Tecnologia e Inovações (MCTI) do Brasil.

Partindo da necessidade de reconhecer a visibilidade, o impacto e as relações temáticas e colaborativas estabelecidas pela pesquisa do INMA, visa-se analisar, ao longo de dez anos (2009-2018), a produção periódica científica vinculada a ele por meio de pesquisadores que, no período, declararam vínculo com o Instituto. Para concretizar 
este objetivo geral, geram-se indicadores de produção e de citação que permitem responder à questão de pesquisa de como se configura essa produção científica no que tange a áreas, temáticas, autores, periódicos, instituições, países mais produtivos, e qual o impacto dessa produção pelas citações recebidas.

Analisar essa atividade possibilita inferir as características da pesquisa que o INMA vem fomentando, de modo a contribuir para a reconstituição histórica de sua memória - a partir dos pesquisadores que por ele passaram, instituições e países com os quais se relacionou mais intensamente e para reconhecer o impacto global dessas pesquisas no âmbito científico, a fim de contribuir com o delineamento futuro das áreas de competência do Instituto nas pesquisas relacionadas à Mata Atlântica.

A relevância deste artigo está associada, também, à necessidade de se organizar e preservar o conhecimento científico produzido e fomentado pelo Instituto e de conhecer suas relações colaborativas como instituição administrada pelo poder público, cuja missão é oportunizar a produção, a preservação e a socialização do conhecimento registrado sobre a Mata Atlântica. Essa missão deve ser evidenciada, em meio à conjuntura de escassez de investimentos em pesquisa científica, para a conservação da biodiversidade da Mata Atlântica brasileira.

\section{REFERENCIAL TEÓRICO}

A aplicação de estudos métricos da informação para mapeamento e análise da produção científica de um domínio científico e/ou institucional é tarefa essencial para a reconstrução da história de instituições e áreas de pesquisa, bem como para o reconhecimento de suas identidades científicas.

Além disso, os estudos métricos da informação, reconhecidos em técnicas bibliométricas e cientométricas, auxiliam diretamente na avaliação da ciência e contribuem para a análise e elaboração de indicadores de desempenho. Acerca do papel dos indicadores, constituem-se como dados estatísticos utilizados para analisar e avaliar as potencialidades da base científica e tecnológica de países (Silva, Sobral, Santana, \& Cruz, 2012).

Os indicadores gerados por meio da aplicação de métricas de informação auxiliam o processo de avaliação de qualquer área do conhecimento e podem responder a questionamentos sobre o crescimento, o declínio e a difusão de determinadas temáticas. Ainda, contribuem com o monitoramento de oportunidades e com a identificação de atividades e projetos mais promissores para o futuro em diferentes áreas, auxiliando nas decisões estratégicas dos gestores de políticas científicas e tecnológicas (Silva et al., 2012, p. 21).

Especialmente nas últimas décadas, o domínio de estudos métricos tem propiciado a geração de indicadores no âmbito do planejamento, gestão, análise e avaliação das políticas de pesquisa em ciência e tecnologia.

Inclusive, tem contribuído com feedbacks sobre os impactos proporcionados pela inovação, subsidiando análises da produção científica em diferentes áreas científicas. A possibilidade de reconhecer padrões de comportamento da produção periódica científica em níveis micro, meso e macro tem sido crucial para a construção de políticas de informação em contextos diversos. Nesse sentido, o campo da Ciência da Informação (CI), por meio dos estudos métricos da Informação, tem como um de seus papéis contribuir para a produção, organização e análise de informação em diferentes domínios do conhecimento, fazendo jus à sua natureza metacientífica.

Cabe aqui a conceituação de Bates (1999) para a Ciência da Informação, para quem a CI é uma metaciência que conduz pesquisas e desenvolve teorias relativas aos produtos de registros da informação de outras disciplinas e atividades. Para a autora, essa metaciência cruza transversalmente diferentes campos do conhecimento, tendo como domínio o universo da informação registrada.

Logo, propiciar o reconhecimento das perspectivas metodológicas e dimensões de análise desenvolvidas pela área, para além dela, é também exercer seu propósito científico. Os estudos que trazem dados e interpretações relativas a indicadores de produção, colaboração, impacto e visibilidade científica têm sido objeto de atenção em distintas áreas e tema de discussão na ciência atual, especialmente no âmbito das políticas científicas.

Em uma tentativa de organizar os tipos de bibliometria - modalidade de estudo métrico — de acordo com suas finalidades, Glänzel (2003) propôs três perspectivas distintas: bibliometria para praticantes da bibliometria (G1); bibliometria aplicada às disciplinas científicas (G2) e bibliometria para a geração de indicadores voltados às políticas científica e de gestão (G3). Os estudos métricos do G2 contribuem também para outras áreas do conhecimento, além da CI, representando a perspectiva mais adequada ao contexto deste artigo. Para Glänzel (2003), esta modalidade de estudos métricos aplicados constitui-se no mais abrangente e diversificado grupo da bibliometria, no qual os interesses estão relacionados com a área de especialidade analisada.

A interação entre pesquisadores de estudos métricos da informação e de outras áreas pode propiciar interessantes e inovadoras linhas de pesquisa. Conforme Meneghini e Packer (2010), com quase nenhuma exceção, os primeiros artigos sobre o tema foram escritos por pesquisadores das ciências naturais, que encontraram uma nova oportunidade para empregar ferramentas quantitativas a fim de criar estímulos à inserção da produção científica brasileira no cenário internacional (Meneghini \& Packer, 2010, p. 2). 
Estudos similares a esse podem ser referenciados no âmbito da CI, especialmente por serem aplicados à área de Ciências Biológicas. É o caso do artigo de Santin, Vanz, e Stumpf (2014), que permite a visualização das redes de colaboração referentes à produção científica de biologia evolutiva dos anos 2000 a 2012. Segundo as autoras, o estudo das interações entre os principais atores de uma área permite revelar padrões e tendências de colaboração nacional e internacional. Outro estudo relevante para o contexto é o de Santin e Silva (2013), no qual destacam a proeminência do campo de biologia evolutiva no contexto nacional e o alto impacto de citação internacional e de índices de colaboração internacional pelas publicações que analisam justificando assim a importância dos estudos métricos aplicados à compreensão de outras áreas.

Já, Lima, Maroldi, e Silva (2012) analisam o comportamento científico do Programa de Mestrado em Biologia da Universidade Federal de Rondônia a partir de suas dissertações. Do mesmo modo, o artigo intitulado "Produção científica em Ciências Biológicas da UFRGS: tendências temáticas no período 2000-2011" apresenta o crescimento da produção e as temáticas evidenciadas no Programa (Santin, Vanz, \& Stumpf, 2015). Ainda nessa linha, Gheno et al. (2020) tratam do impacto da internacionalização na visibilidade da produção científica do Programa de Pós-Graduação em Ciências Biológicas: BIOQUÍMICA/UFRGS (2007-2016).

Outro estudo similar é o de Silva e Brisola (2017), no qual são mapeadas as publicações de docentes da PósGraduação em Biologia Molecular e Celular da Universidade Federal do Estado do Rio de Janeiro, de 2011 a 2016, a fim de propiciar maior conhecimento sobre o perfil da pesquisa desse grupo e traçar ações que favoreçam a melhoria da pontuação do referido Programa de Pós-Graduação. Os autores apresentam, na publicação, os tipos documentais com maior índice de publicação, o percentual de pesquisadores mais produtivos e o perfil de periódicos publicadores, quantificando os esforços para a ampliação da visibilidade internacional da produção brasileira.

Silveira e Teixeira (2017), em "Conhecimento compartilhado em rede: um estudo da área de Ciências Biológicas e Ciências Ambientais do IFRS, campus Porto Alegre (RS)" desvelam as práticas de pesquisa e a produção acadêmica dos docentes efetivos e dos colaboradores da área de Ciências Biológicas e Ciências Ambientais da referida Instituição, por meio da cientometria.

Segundo os autores, atuar em redes de colaboração permite a ampliação e a aplicação de sua produção científica de forma mais substancial, visto que a interação de conhecimentos e competências acelera a geração de inovações.

O referencial apresentado demonstra que os estudos métricos da informação aplicados à área de Biologia vêm sendo amplamente utilizados e voltam-se tanto à gestão de políticas científicas, como ao delineamento de perfis de atividades de pesquisa.

\section{MÉTODO}

Este artigo parte dos estudos métricos da informação para gerar indicadores de produção e de impacto da produção periódica científica de artigos vinculada ao INMA pela afiliação institucional de seus autores em um recorte temporal de dez anos (2009-2018).

A consolidação do corpus de análise foi realizada com base na produção de 41 pesquisadores vinculados ao INMA (mapeados a partir de análises de documentos institucionais administrativos), cujos artigos publicados nos períodos de vigência dos seus vínculos com o Instituto estão indexados na base de dados Scopus. Totalizou-se um corpus de 73 artigos no período de 2009 a 2018. O corpus obtido da Scopus foi exportado para a base de dados Scival (também da Elsevier) que contribuiu como instrumento de geração e análise de indicadores.

Identificaram-se as áreas de concentração da produção delimitada, as temáticas dos artigos por meio de suas palavras-chave, os periódicos publicadores de destaque no domínio institucional, os anos de maior produtividade e de impacto da produção e se reconheceu a colaboração por meio de autores, instituições e países colaboradores que se destacaram pelos indicadores de produtividade.

A nuvem de palavras-chave, construída na base Scival, considerou as 50 palavras-chave mais incidentes do corpus, apresentando as palavras em crescimento, as que se mantiveram estáveis e as que diminuíram a incidência no decorrer do período analisado.

É importante ressaltar que os indicadores utilizados para a mensuração do impacto da produção analisada foram: a) quantidade de citações recebidas pelos artigos na Scopus; b) indicador Field-Weighted Citation Impact - FWCI), visualizado na Figura 2, que é um indicador de citação ponderada nas áreas de concentração dos artigos na base Scopus, ou seja, ele mede a proporção do total de citações que foram recebidas pela saída do denominador e o total de citações esperadas, com base na média de citação do referido campo de assunto. O valor deste indicador, quando é igual a 1, indica que o artigo obteve um desempenho dentro da expectativa para os artigos daquela área de concentração; quando é maior do que 1, indica que o artigo é mais citado que o esperado para a média global no campo de assunto, por exemplo: um artigo que apresente 1,60 de FWCI, obteve $60 \%$ mais citações do que a média global; quando o valor é menor que 1, indica que o artigo é menos citado que a média global de citação no campo de assunto daquele artigo. Finalmente um último indicador utilizado foi: c) 
Citations per document (2 years) para mensuração do impacto e visibilidade dos periódicos que, no intuito de simplificar sua escrita, denominou-se como índice de impacto. Este indicador foi coletado no Scimago Journal \& Country Rank (Scopus) e equivale ao Fator de Impacto (FI) da base de dados Web of Science da Clarivate Analytics.

A produção enfocada limita-se à pesquisa de maior visibilidade e impacto vinculada ao INMA, excluindo assim, a diversidade de publicações vinculadas ao Instituto que não correspondem à literatura mainstream indexada na Scopus. Em contrapartida, o corpus permite obter um retrato dos estudos que se constituem como elemento de identidade e referência internacional da pesquisa consignada ao INMA, a partir de seus colaboradores.

Vale destacar que as colaborações do INMA que não se caracterizam como coautorias, mas que originam menções ao Instituto nos campos de busca de agradecimentos e financiamentos, foram mapeadas anteriormente à esta análise, em estudo exploratório na Scopus, mas inexpressivas quantitativamente para fins de análise. Por essa razão, optou-se pelo mapeamento das pesquisas produzidas pelos pesquisadores no período de vínculo com o INMA. Também se contemplou na análise os colaboradores voluntários que se autodeclaram como pesquisadores do INMA.

\section{RESULTADOS E DISCUSSÃO}

O INMA foi descrito como afiliação institucional de autores publicadores de 73 artigos, no período de 2009 a 2018, na base de dados Scival. Esse corpus envolveu 385 autores e também recebeu 308 citações nesses dez anos, uma média de 4,22 citações por artigo. Apenas 15,6\% dos artigos (11) do corpus não receberam citações, o que indica que essa produção apresenta impacto expressivo.

A produção por áreas do conhecimento definidas pela Scopus, para o corpus em questão, ficou assim distribuída: a maioria dos artigos do corpus está concentrada em "Agricultural and Biological Sciences", perfazendo um total de 69 artigos consignados a este domínio, o que representa 84,1\% do conhecimento socializado em artigos científicos e artigos de revisão.

A segunda maior área de concentração é a "Environmental Science" (8,5\%) com um total de sete artigos indexados. Em seguida, na terceira posição, aparece o domínio de "Biochemistry, Genetics and Molecular Biology", com três artigos (3,7\%). Já "Earth and Planetary Sciences" foi representada por 2,4\% da produção (dois artigos) e, finalmente, "Veterinary" teve apenas um artigo indexado $(1,2 \%)$.

A Tabela 1 mostra como as publicações foram subdivididas, levando em conta as subáreas da grande área "Agricultural and Biological Sciences". Considerando que um artigo pode ser contemplado em mais de uma área e/ou subárea, a subárea mais contemplada foi a de "Ecology, Evolution, Behavior and Systematics" com 39,1\% da produção, totalizando 43 artigos publicados no período. A subárea "Plant Science" ficou com a segunda maior concentração, com 27,3\% da produção em um total de 30 artigos produzidos. A terceira maior concentração de artigos foi na subárea "Animal Science e Zoology" com 22 artigos (20\%). As demais subáreas ficaram assim atendidas: "Aquatic Science" com nove artigos (8,2\%), "Horticulture" com três publicações (2,7\%) e outras três publicações 2,7\% sem classificação especificada pela base de dados Scival. Essa configuração pode evidenciar linhas de pesquisa mais atuantes do INMA no período retratado. 


\begin{tabular}{|c|c|c|}
\hline \multicolumn{3}{|l|}{ Agricultural and Biological Sciences $(84,1 \%)$} \\
\hline Subárea & $(\%)$ & $n^{\circ}$ artigos \\
\hline Ecology, Evolution, Behavior and Systematics & 39,1 & 43 \\
\hline Plant Science & 27,3 & 30 \\
\hline Animal Science and Zoology & 20,0 & 22 \\
\hline Aquatic Science & 8,2 & 9 \\
\hline Horticulture & 2,7 & 3 \\
\hline Other & 2,7 & 3 \\
\hline \multicolumn{3}{|l|}{ Environmental Science (8,5\%) } \\
\hline Subárea & (\%) & $n^{\circ}$ artigos \\
\hline Ecology & 55,6 & 5 \\
\hline Ecological Modeling & 11,1 & 1 \\
\hline Environmental Chemistry & 11,1 & 1 \\
\hline General Environmental Science & 11,1 & 1 \\
\hline Nature and Landscape Conservation & 11,1 & 1 \\
\hline \multicolumn{3}{|l|}{ Biochemistry, Genetics and Molecular Biology (3,7\%) } \\
\hline Subárea & (\%) & $n^{\circ}$ artigos \\
\hline Genetics & 40 & 2 \\
\hline Molecular biology & 40 & 2 \\
\hline General Biochemistry, Genetics and Molecular and Molecular Biology & 20 & 1 \\
\hline \multicolumn{3}{|l|}{ Earth and Planetary Sciences $(2,4 \%)$} \\
\hline Subárea & (\%) & $n^{\circ}$ artigos \\
\hline Atmospheric Science & 50 & 1 \\
\hline Oceanography & 50 & 1 \\
\hline \multicolumn{3}{|l|}{ Veterinary } \\
\hline Subárea & (\%) & $n^{\circ}$ artigos \\
\hline General veterinary & 100 & 1 \\
\hline
\end{tabular}

Tabela 1. Distribuição da produção científica analisada nas categorias do conhecimento da Base de dados Scival (2009-2018) Fonte: Dados da pesquisa (2020).

A ecologia como especialidade da biologia que estuda os seres vivos, o meio ambiente e a interação entre eles abarca uma diversidade de outras áreas de estudo, permitindo o entendimento do funcionamento global da natureza, sua conservação e biodiversidade. A representatividade numérica dos artigos afiliados ao INMA na subárea "Ecology" pode ser explicada pela amplitude temática que esse domínio científico congrega e, além disso, pelo foco da pesquisa do INMA, no conhecimento e conservação da biodiversidade da Mata Atlântica.

Já que uma única publicação pode se adequar a mais de uma área temática, os estudos relativos à fauna e flora também podem ser contemplados pelo domínio da ecologia, quando visam estudar a interação desses seres com o meio ambiente ou entre eles. Isso pode explicar o expressivo percentual de estudos na subárea de Agricultural and Biological Sciences denominada "Ecology, Evolution, Behavior and Systematics" e na subárea de "Environmental Science", denominada "Ecology".

Sugere-se que um dos motivos para o destaque de "Plant Science" como domínio de publicação, nos dez anos analisados, decorre da presença de um dos autores mais produtivos do corpus no INMA neste período como voluntário e bolsista, publicando sobre as famílias botânicas retratadas na Figura 1.

Deve-se considerar a importância histórica que a coleção de botânica do Herbário MBML apresenta desde sua fundação. Segundo Bates (1959), o Herbário foi fundado em 1940 por Augusto Ruschi, com o número de 3 mil espécimes. Nos últimos 30 anos, o Herbário apresentou incremento exponencial no número de exemplares, devido à intensa atividade de campo e coletas realizadas em parcerias e projetos com pesquisadores oriundos de distintas instituições, como por exemplo o projeto do CNPq (400360/01-2) "Biodiversidade da Mata Atlântica do Espírito Santo" que serviu de apoio para as pesquisas realizadas no INMA na década de 2000, no qual Ludovic Kollman, que é o autor mais produtivo do corpus, integrou. Outros projetos como o Projeto Cores, vinculado ao Instituto de Pesquisas do Jardim Botânico do Rio de Janeiro destaca as parcerias institucionais do INMA, que geraram pesquisas em coautoria.

O reconhecimento do Herbário MBML no número 11 do Boletim do Instituto Paranaense de Botânica de 1959, por Angely, contribuiu para sua visibilidade para pesquisa nacional. Do mesmo modo, sua indexação no Index Herbariorum do Jardim Botânico de Nova York (New York Botanical Garden, 2018), por atender a critérios mínimos de volume da coleção, permitiu que o mesmo ganhasse notoriedade internacional. Soma-se aí a informação de que as visitas externas à coleção botânica ali depositada se aproximaram de 957 consultas no 
período dos dez anos analisados. Sugere-se que esses eventos podem ter contribuído, mesmo que de modo indireto, para o reconhecimento do Herbário como meio de pesquisa por pesquisadores externos, gerando parcerias em publicações.

É importante destacar que, embora a área de concentração "Plant Science", correspondente à botânica, apresente maior quantidade de artigos publicados, não corresponde à área de maior impacto de citação, que no corpus foi a "Animal Science and Zoology". Observa-se que três, dos cinco artigos de maior impacto de citação do corpus, são relacionados ao domínio da herpetologia, que estuda anfíbios e répteis.

Quanto às subáreas da grande área "Environmental Science" (Tabela 1), a maior concentração de artigos também foi em "Ecology", com 55,6\% dos artigos indexados nessa subárea, totalizando cinco publicações. As demais subáreas "Ecological Modeling", "Environmental Chemistry", "General Environmental Science" e "Nature and Landscape Conservation", tiveram concentração de 11,1\%, o equivalente a um artigo cada.

A área "Biochemistry, Genetics and Molecular Biology", com 3,7\% das publicações indexadas, abarcou três subáreas: "Genetics" e "Molecular Biology", que concentraram 40\% (duas publicações) cada; e "General Biochemistry, Genetics and Molecular Biology" com $20 \%$ da produção (uma publicação). "Earth And Planetary Sciences" como demonstrado na Tabela 1 abarcou duas subáreas, com 50\% cada uma (um artigo) do total de artigos indexados, sendo elas "Atmospheric Science" e "Oceanography". E, finalmente, a área "Veterinary", teve apenas um artigo indexado (1,2\%) na subárea "General Veterinary".

Identificam-se, também, temáticas estudadas por meio das palavras-chave dos artigos. A nuvem de palavras (Figura $1^{1}$ ) foi construída com base nas 50 palavras-chave mais utilizadas nos artigos do corpus. As palavras em verde estão em crescimento, as de cor cinza estão estabilizadas e as azuis declinaram, caindo no desuso no decorrer do tempo.

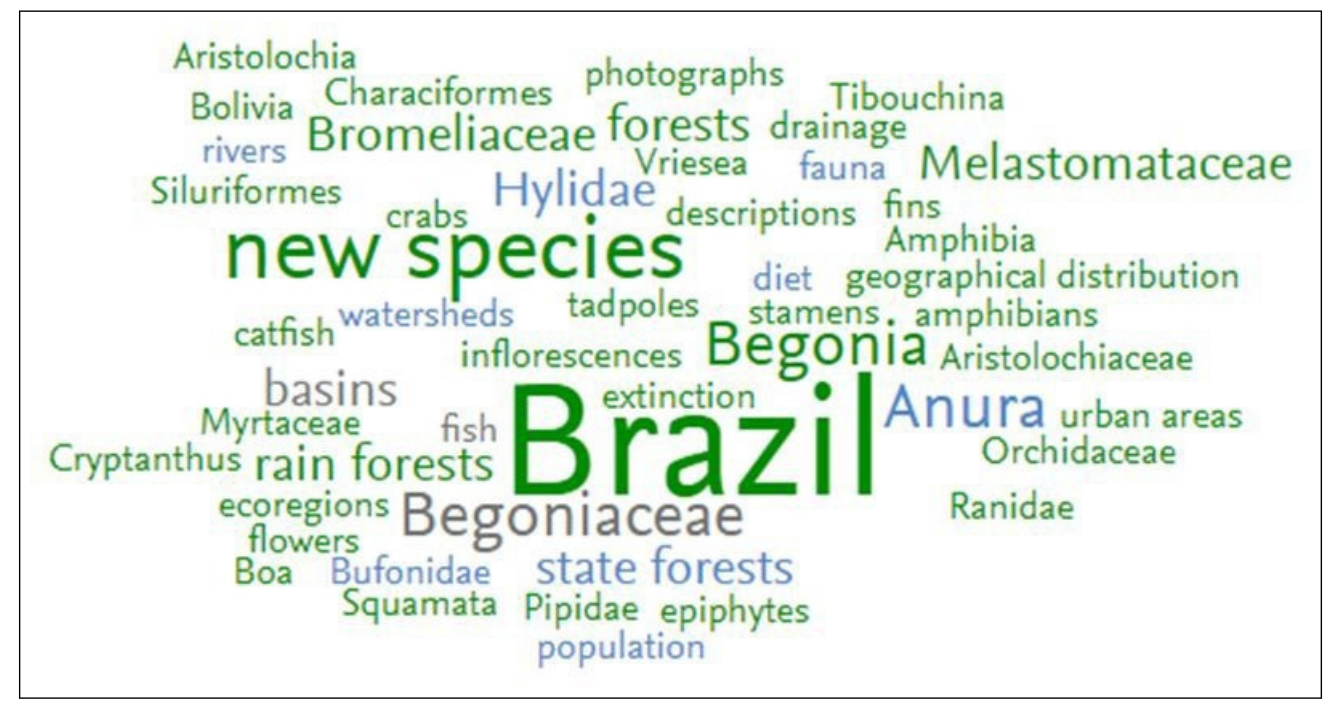

Figura 1. Nuvem de palavras-chave da produção científica afiliada aos pesquisadores do INMA entre 2009 e 2018. Fonte: Os autores (2020).

Observa-se que os estudos sobre novas espécies se destacam significativamente, devido ao crescimento do uso do termo no período analisado. Na área da botânica, observa-se o crescimento do uso de várias palavras-chave, como Aristolochia, Tibouchina, Vriesea, stamens, inflorescences, Aristolochiaceae, Myrtaceae, Cryptanthus, Orchidaceae, flowers e epiphytes. Algumas famílias botânicas como Melastomataceae e Bromeliaceae evidenciamse nas temáticas crescentes para o período. Por outro lado, aparentemente a palavra-chave Begoniaceae, que representa uma família botânica, deu lugar ao uso do termo Begonia, que representa um gênero dessa família. Na área da zoologia, também se observa o crescimento do uso de várias palavras-chave, com destaque para o domínio da ictiologia (estudo dos peixes), denotado por termos como Characiformes, Siluriformes e catfish, bem como da herpetologia (estudo dos anfíbios e répteis), com destaque para os termos Amphibia, tadpoles, amphibians, Ranidae, Pipidae, Boa e Squamata. Os termos que remetem ao ambiente, em geral, estão relacionados a estudos de ecologia ou conservação, como forests, drainage, extinction, urban areas, rain forests, ecoregions.

Considerando que os pesquisadores colaboradores do Instituto são, em sua maioria, transeuntes/temporários, e em pequeno número, conforme será evidenciado no decorrer do texto, observa-se que o perfil temático das publicações é fortemente influenciado pelo período em que permanecem no Instituto.

\footnotetext{
${ }^{1}$ As palavras em verde estão em crescimento, as de cor cinza estão estabilizadas e as azuis declinaram.
} 


\section{Quanto ao impacto e visibilidade}

Sobre a visibilidade e o impacto das pesquisas do corpus, pode-se dizer que entre 2009 e 2018 o INMA socializou sua produção científica em 39 periódicos, sendo que, destes, $72 \%$ são internacionais, o que demonstra elevada internacionalização das pesquisas filiadas aos pesquisadores do Instituto.

A seguir visualiza-se a distribuição dos artigos do corpus no decorrer dos dez anos, bem como o impacto de citação desses artigos e seus índices de proeminência relacionados às temáticas tratadas. Esses elementos baseiam-se em indicadores provenientes da base Scopus, visualizados dentro da base Scival.

No gráfico que representa a distribuição das publicações, os anos de 2010 e de 2016 foram marcados pelo ápice de produtividade das pesquisas do corpus, e os anos de 2016 e 2017 apresentam elevado número de citação. Vale ressaltar que o impacto de citação de uma publicação só pode ser apropriadamente mensurável após dois anos de sua publicação. Observa-se uma distribuição e crescimento irregular da produção no período analisado. Essa irregularidade converge com a conjuntura do INMA, atípica para uma unidade de pesquisa pública brasileira, que conta apenas com $7 \%$ de pesquisadores com vínculos efetivos de servidores públicos, sendo, o restante, bolsistas e voluntários que desenvolvem pesquisas de modo temporário.

O índice de proeminência temática dos artigos apresenta crescimento mais expressivo em 2016, tendo seu pico nos artigos publicados em 2017, com proeminência de 0,77 para os estudos do corpus (esse indicador vai de 0 a $1)$.

Considera-se que nem sempre a expressividade quantitativa de artigos é proporcional ao impacto e à proeminência desses no meio científico, como é o caso da distinção entre áreas de botânica e zoologia no corpus, em que uma apresenta maior produção e outra se destaca pelo maior impacto. Essa informação pode estar relacionada à quantidade de autores nos artigos. Na botânica há uma menor quantidade de autores no corpus, sendo que o pesquisador que se destaca na primeira posição como autor mais produtivo (Tabela 4) é da botânica e apresenta $21,7 \%$ dos seus artigos em autoria individual.

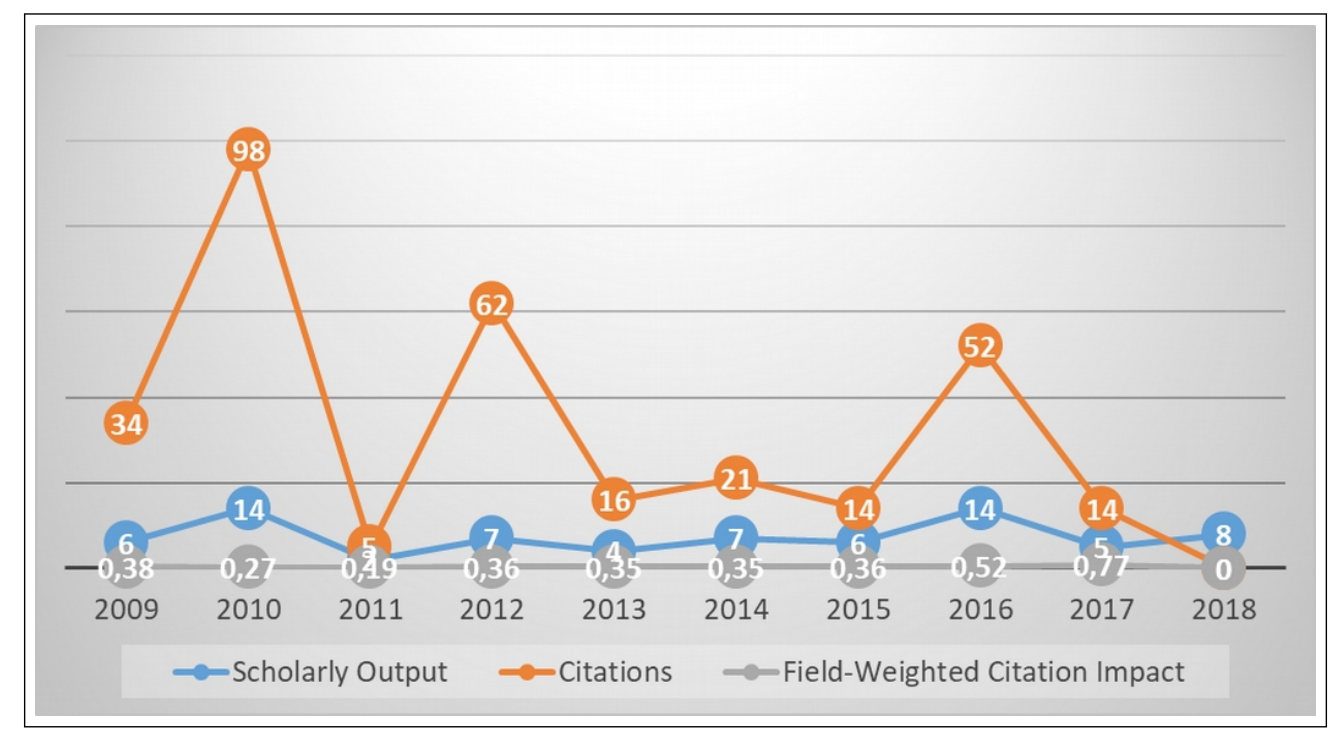

Figura 2. Distribuição, impacto e proeminência temática de artigos do corpus no período. Scholarly Output: número de artigos indexados na Scopus; Citations: número de citações recebidas na Scopus; Field Weighted Citation Impact: média global de impacto de citação na área indexada na Scopus.

Fonte: Os autores (2020).

A produção analisada, embora pequena para os padrões institucionais, tem impacto de citação e visibilidade internacional superior ao esperado, como se pode verificar na quantidade de citações recebidas pelos artigos afiliados em cada periódico, e também, pelo índice de impacto dos próprios periódicos publicadores (Tabela 2).

Os cinco periódicos mais utilizados para publicação pelo INMA foram: Candollea (oito artigos), Phytotaxa (sete artigos), Herpetology Notes (seis artigos), Check List (quatro artigos) e Pan-American Journal of Aquatic Sciences (quatro artigos).

A Tabela 2 apresenta os sete periódicos dentre os 39 com maior visibilidade mensurada pela quantidade de citações recebidas (mais de 20 citações cada) no período analisado. O periódico que trouxe significativa visibilidade ao Instituto no período analisado foi Phytotaxa, da Nova Zelândia. Neste, o INMA publicou sete artigos (segundo periódico mais utilizado para socialização do conhecimento), os quais receberam 31 citações, perfazendo uma média de 4,43 citações por artigo. Essa média é expressivamente maior que a expectativa de impacto do periódico mensurada pelo indicador "Citations per document" do Scimago Journal Country Ranks (2020), que aqui 
chama-se de índice de impacto, que para este periódico foi de 1,232. Ressalta-se que este indicador é equivalente ao Fator de Impacto da base de dados Web of Science.

\begin{tabular}{l|c|c|c|c}
\hline Revista & País & índice de impacto & Citações recebidas & Total de artigos \\
\hline Phytotaxa & Nova Zelândia & 1,232 & 31 & 7 \\
Rodriguesia & Brasil & 0,743 & 29 & 3 \\
Journal of Herpetology & Estados Unidos & 1,030 & 27 & 1 \\
Candollea & Suíça & 0,606 & 23 & 8 \\
Pan-American Journal of & Brasil & 0,493 & 23 & 4 \\
Aquatic Sciences & Alemanha & 0,975 & 22 & 6 \\
Herpetology Notes & Reino Unido & 0,731 & 21 & 3 \\
Kew Bulletin &
\end{tabular}

Tabela 2. Impacto e visibilidade dos periódicos utilizados pelos pesquisadores afiliados ao INMA entre 2009 e 2018. Fonte: Dados da pesquisa (2020).

Outros seis periódicos, com mais de 20 citações, oportunizam visibilidade ao Instituto, sendo dois deles brasileiros, o que pode sugerir uma expressiva visibilidade internacional do conhecimento socializado em periódicos brasileiros. É o caso de Rodriguesia, do Jardim Botânico do Rio de Janeiro, com índice de impacto 0,743, que proporcionou ao INMA 29 citações em três artigos publicados no período, em uma média de 9,67 citações por artigo. Na terceira posição, quanto à visibilidade mensurada pela quantidade de citações recebidas, aparece o periódico Journal of Herpetology (índice de impacto 1,030) dos EUA, que obteve 27 citações em apenas um artigo. Ressalta-se que esse artigo intitulado "Diet, fecundity, and use of bromeliads by Phyllodytes luteolus (Anura: Hylidae) in southeastern Brazil" da autoria de autores mais produtivos destacados na Tabela 4, foi o que mais recebeu citações no período analisado, tendo como coautores dois dos pesquisadores evidenciados no corpus, e também, como instituição parceira, a Utah State University.

Os periódicos Candollea da Suíça (índice de impacto 0,606) com oito artigos (periódico que mais veiculou publicações do INMA), e Pan-American Journal of Aquatic Sciences do Brasil (índice de impacto 0,493), com quatro artigos, receberam 23 citações cada no período analisado, seguidos dos periódicos Herpetology Notes (índice de impacto 0,975) da Alemanha (com seis artigos) e Kew Bulletin (índice de impacto 0,975) do Reino Unido (com três artigos) que receberam 22 e 21 citações respectivamente.

Os sete periódicos anteriormente citados, que proporcionaram maior visibilidade ao INMA, captaram cerca de $56,4 \%$ do total de citações recebidas. Vale ressaltar também, que estes sete periódicos foram responsáveis por socializar cerca de $44 \%$ do total produzido pelo Instituto na década analisada.

Denota-se que seus escopos estão diretamente relacionados à especialidade dos autores que mais publicaram e com as temáticas constantes nas palavras-chave. O Rodriguesia, periódico brasileiro de maior visibilidade no corpus, tem como escopo estudos relacionados aos diversos domínios da biologia vegetal, bem como da história da botânica e as atividades ligadas aos jardins botânicos (Rodriguesia, 2020).

Por meio do escopo dos periódicos, é notório que a botânica e a zoologia, mais especificamente com a herpetologia, se destacam no recorte temporal analisado.

\section{Quanto à colaboração em nível micro, meso e macro (autorias, instituições e países)}

No que concerne à colaboração institucional, mensurada pelas afiliações dos coautores, a Tabela 3 apresenta as cinco instituições que mais colaboraram com o INMA no período de 2009 a 2018. As três primeiras instituições colaboraram com grande parte da produção do INMA, resultado expressivo que demonstra laços estreitos entre elas.

\begin{tabular}{lc}
\hline Instituição colaboradora & $\begin{array}{c}\text { Quantidade } \\
\text { de artigos }\end{array}$ \\
\hline Universidade Federal do Rio de Janeiro & 19 \\
Universidade Federal do Espírito Santo & 13 \\
Utah State University & 11 \\
Instituto de Pesquisas Jardim Botânico do Rio de Janeiro & 8 \\
Universidade Vila Velha & 7 \\
\hline
\end{tabular}

Tabela 3. TOP 5 das instituições que mais publicaram artigos em coautoria com o INMA entre 2009 e 2018.

Fonte: Dados da pesquisa (2020).

A principal instituição colaboradora, com 19 artigos, com mais de $1 / 4$ de toda a produção do INMA no período analisado (26\% do total) foi a Universidade Federal do Rio de Janeiro (UFRJ). De acordo com o Ranking 
Universitário Folha (RUF), 2019, a UFRJ é considerada a terceira melhor instituição de ensino superior do país, ficando atrás apenas da Universidade de São Paulo (USP) e da Universidade Estadual de Campinas (Unicamp). Já no The world university rankings 2020 (2020) a UFRJ está posicionada entre as 801-1.000 primeiras.

A Universidade Federal do Espírito Santo (UFES) aparece como a segunda maior colaboradora do INMA, com 13 artigos (18\% do total produzido) e ocupa a $27^{\mathrm{a}}$ posição no RUF e também, posição acima de 1.001 no ranking THE. Entre as cinco instituições que mais colaboraram com o INMA observa-se a Utah State University como única instituição estrangeira, que ocupa posição no grupo 201-250 das melhores universidades do mundo no Times Higher Education (THE), e com a qual o INMA obteve parceria em 15\% dos artigos (11 publicações).

O Instituto de Pesquisas Jardim Botânico do Rio de Janeiro, que ocupa a posição 839 no Ranking Web of Research Centers (2019), aparece na quarta posição entre as cinco instituições com maior colaboração com o INMA. Com esta instituição o INMA publicou 8 artigos (10,9\%). Essa relação de colaboração não necessariamente explicita a estreita e intensa proximidade entre pesquisadores de ambas instituições, que trabalharam em conjunto com a coletas botânicas em projetos distintos, transcendendo assim, os tipos de colaboração que não somente por coautorias.

Por último, na quinta posição das instituições mais presentes no corpus, encontra-se a Universidade Vila Velha (UVV-ES) (112 $2^{\mathrm{a}}$ posição no RUF), com quem o INMA publicou em parceria 9,5\% de sua produção entre 2009 e 2018. Ressalta-se que a forte presença da UFES e da UVV-ES pode ser explicada devido à proximidade geográfica, o que facilita as colaborações institucionais entre INMA e instituições de ensino superior, as quais se beneficiam da estrutura de pesquisa do Instituto para realização de suas coletas, consultas, depósitos e aulas de campo.

Quanto ao país de origem dos pesquisadores coautores da produção científica do INMA, dentre os cinco que mais colaboraram encontra-se um país da América do Norte, dois da América Latina e dois Europeus.

Os Estados Unidos, representante da América do Norte, é o maior detentor da produção científica mundial, sendo primeiro colocado do Scimago Journal Country Rank (SJR) com Índice $\mathrm{h}=2222$. O referido país teve seus pesquisadores como coautores em $17,8 \%$ de toda produção científica do INMA no período analisado. Sobre os demais países, cada um contribuiu em 3 artigos (4,1\%), a saber: como representantes da América Latina — a Colômbia como segundo país que mais colaborou com o INMA, ocupando atualmente a $50^{\mathrm{a}}$ posição no SJR da Scopus, com Índice $\mathrm{H}=261$ e a Argentina, como quinto país que mais colaborou com o INMA, ocupando a $37^{\mathrm{a}}$ posição no SJR com Índice $\mathrm{H}=393$. Como representantes da Europa, a Alemanha é o terceiro país que mais colaborou com o INMA e ocupa a $4^{\mathrm{a}}$ posição no SJR com Índice H $=1203$ e o Reino Unido é o quarto país que mais colaborou com o INMA e ocupa a $3^{\text {a }}$ posição no SJR com Índice $H=1373$.

As colaborações identificadas pela coautoria com Estados Unidos, Reino Unido e Alemanha se consolidam prioritariamente no domínio da zoologia. Já as colaborações com países da América do Sul apresentam-se, principalmente, na botânica.

Deve-se reconhecer que a colaboração científica que o INMA proporciona vai além das coautorias de seus pesquisadores afiliados, visto que o Instituto é um espaço, lócus de conhecimento científico, com coleções biológicas ali alocadas, fato que o torna um agente importante no fomento à pesquisa científica relacionada à Mata Atlântica.

Há distinção entre práticas de colaboração científica e de coautoria de acordo com os domínios do conhecimento. No caso do INMA, a identificação das coautorias permite aferir o corpo de colaboradores institucionais com mais precisão.

Segundo Hilário e Grácio (2017), a média de colaboração por coautoria em artigos na grande área de Ciências Biológicas é, em geral, de 5,3 autores. Contudo, nesse corpus a média é de 3,87 autores por artigo e a média de colaboradores por artigo, referente aos autores mais produtivos, é ainda menor, variando entre 1,5 e 3 autores. Essa informação indica uma maior liderança no desenvolvimento das pesquisas por parte dos autores mais produtivos que estiveram vinculados ao INMA entre 2009 e 2018.

É importante ressaltar que um dos artigos do corpus, datapaper com 218 autores, foi desconsiderado para essa média de colaboração a fim de não enviesar o resultado. Destaca-se que, tanto a visibilidade quanto a probabilidade de citação das pesquisas, é incrementada quando são desenvolvidas em autoria múltipla, especialmente quando se congregam distintas instituições de pesquisa internacionais.

Além disso, a ordem de autoria pode denotar graus de participação de autores. Observa-se que, em 34 artigos do corpus, há primeiros autores afiliados ao INMA, o que representa 47,22\% do total de artigos e mostra uma liderança da pesquisa por parte do Instituto. Indicia-se que o INMA lidera pesquisas e congrega a colaboração de pesquisadores de outras instituições nos domínios de botânica, especialmente com o estudo das bromélias, begônias e melastomatáceas, e de zoologia com o estudo da herpetologia (anfíbios/répteis), e com expressividade mais discreta para a ictiologia (peixes). 


\begin{tabular}{lcc}
\hline Autor & $\begin{array}{c}\text { Quantidade } \\
\text { de artigos }\end{array}$ & $\begin{array}{c}\text { Média de } \\
\text { colaboradores }\end{array}$ \\
\hline Kollmann, Ludovic Jean Charles & 23 & 1,96 \\
Ferreira, Rodrigo Barbosa & 14 & 2,86 \\
Silva-Soares, Thiago & 10 & 2,90 \\
Sarmento-Soares, Luisa Maria & 10 & 2,40 \\
Martins-Pinheiro, Ronaldo Fernando & 7 & 3,00 \\
Teixeira, Rogério Luiz & 7 & 2,43 \\
\hline
\end{tabular}

Tabela 4. TOP 6 dos pesquisadores filiados ao INMA que mais publicaram artigos entre 2009 e 2018. Fonte: Dados da pesquisa (2020).

Em relação aos autores destacados no corpus como filiados ao INMA, Ludovic Jean Charles Kollman, do domínio da botânica, destacou-se em primeira posição devido ao número de publicações. O autor fez trabalho voluntário no herbário MBML na década de 1980 pela primeira vez, trabalhando especialmente com begônias, bromélias e orquídeas. Kollman também contribuiu com pesquisas como bolsista do primeiro Programa de Capacitação Institucional do INMA (PCI/INMA - 2014-2018), estabelecendo diferentes parcerias em seus estudos.

Já Luisa Maria Sarmento Soares, da zoologia (ictiologia), além de pesquisadora voluntária foi bolsista da Fundação de Amparo à Pesquisa do Estado do Espírito Santo (FAPES) no INMA e foi uma das bolsistas do referido PCI-INMA. Também trouxe contribuições para o processo de organização das coleções de zoologia do Instituto.

Outro autor destacado no corpus, Rodrigo Barbosa Ferreira, atua na zoologia (herpetologia) e esteve vinculado ao Department of Wildland Resources and Ecology Center da Utah University, no ano de 2014, instituição mencionada no ranking das cinco instituições mais produtivas do corpus, sendo uma das razões que explica a presença da mesma entre as instituições parceiras.

Thiago Silva-Soares, também atuante na herpetologia, destaca-se entre os cinco pesquisadores mais incidentes do corpus, integrando o Laboratório de Herpetologia do Museu Nacional do Rio de Janeiro. Ambos, Thiago Silva-Soares e Rodrigo Barbosa Ferreira, foram coautores em publicação com pesquisador do Laboratório de Ecologia Terrestre e Aquática da Universidade de Vila Velha. Os dois autores foram bolsistas do Programa PCI.

As variáveis analisadas relativas à produtividade, ao impacto e à visibilidade das pesquisas afiliadas ao INMA, quando contextualizadas historicamente, revelam elementos de identidade do Instituto e contribuem para a preservação de sua memória como objeto de pesquisa científica. Ainda, os resultados podem ser futuramente comparados aos indicadores de produção científica nacional sobre a Mata Atlântica, permitindo inferir se os domínios temáticos estão alinhados e convergentes.

Esta análise, além de configurar-se como parte da memória científica do INMA, contribui como instrumento de gestão institucional, ao apontar as linhas de maior consolidação e mostrar que a visibilidade da pesquisa do INMA é influenciada pelos estudos de bolsistas e voluntários, ou seja, de colaboradores com vínculos temporários.

\section{CONCLUSÕES}

A internacionalização e o impacto das pesquisas vinculadas ao INMA são expressivos, especialmente pelo fato de que os artigos mais citados do corpus apresentam impacto acima da média geral dos seus periódicos publicadores. Somam-se a esse indício de internacionalização, o elevado percentual de revistas internacionais em que o INMA publica. Além disso, há elevada quantidade de citações de artigos, sendo que alguns desses artigos apresentam expressiva proeminência internacional quando comparados aos artigos da mesma área produzidos em âmbito mundial.

Por fim, é possível que o corpus de publicações afiliadas ao INMA seja maior do que o retratado, visto que nem todas as pesquisas realizadas com a colaboração do INMA como instituição afiliada vêm sendo creditadas adequadamente ao Instituto como instituição primária ou secundária de afiliação e/ou com nomenclaturas padronizadas que possibilitem uma recuperação exaustiva.

Isso pode servir de alerta para que as afiliações de autores sejam declaradas adequadamente pelos pesquisadores enquanto colaboradores de uma instituição, a fim de que a produção periódica científica decorrente das parcerias possa ser mapeada exaustivamente, oportunizando, assim, análises acuradas sobre o caráter colaborativo das pesquisas de uma instituição e um retrato mais fidedigno de sua memória. 


\section{AGRADECIMENTOS}

À Doutora Maria Cláudia Cabrini Grácio, pelos direcionamentos que contribuíram para o aperfeiçoamento deste estudo. 


\section{REFERÊNCIAS}

Bates, M. J. (1959). Instituições de botânica do brasil: index herbariorum. Boletim do Instituto Paranaense de Botânica(11), 1-39.

Bates, M. J. (1999). The invisible substrate of information science. Journal of the American Society for Information Science, 50(12), 1043-1050.

Gheno, E. M., Vanz, S. A. d. S., Martins, L. A. M., Duarte, L. F., Souza, D. O., \& Calabró, L. (2020). Impacto da internacionalização na visibilidade da produção científica do programa de pós-graduação em ciências biológicas: Bioquímica/ufrgs (2007-2016). Encontros Bibli: Revista Eletrônica de Biblioteconomia e Ciência da Informação, 25. doi: 10.5007/1518-2924.2019.e65382.

Glänzel, W. (2003). Bibliometrics as a research field: a course on theory and application of bibliometric indicators. Recuperado de https://www.researchgate.net/publication/ 242406991 Bibliometrics_as_a_research_field_A _course_on_theory_and_application_of_bibliometric - indicators

Hilário, C. M., \& Grácio, M. C. C. (2017). Scientific collaboration in brazilian researches: a comparative study in the information science, mathematics and dentistry fields. Scientometrics, 113(2), 929-950.

Lima, L. F. M., Maroldi, A. M., \& Silva, D. V. O. (2012). Análise de citações em literatura inglesa nas dissertações do programa de mestrado em biologia da universidade federal de rondônia. In Iv encontro brasileiro de bibliometria $e$ cientometria. Gramado, Brasil.

Meneghini, R., \& Packer, A. L. (2010). The extent of multidisciplinary authorship of articles on scientometrics and bibliometrics in brazil. Interciencia, 35(7), 510-514.

New York Botanical Garden. (2018). Index herbariorum. Recuperado de http://sweetgum.nybg.org/science/ih/.

Rodriguesia. (2020). Rodriguesia. Recuperado de https:// rodriguesia.jbrj.gov.br.

Santin, D. M., \& Silva, R. C. P. d. (2013). Internacionalização da produção científica brasileira em biologia evolutiva: 2000-2012. In Xiv encontro nacional de pesquisa em ciência da informação. Florianópolis, Brasil. Recuperado de http:// repositorios.questoesemrede.uff.br/repositorios/bitstream/ handle/123456789/2454/INTERNACIONALIZA\%c3\% $87 \%$ c3\%83O $\% 20$ DA $\% 20$ PRODU $\%$ c3\%87\%c3\%830\% 20CIENT\%c3\%8dFICA.pdf? sequence $=1$.

Santin, D. M., Vanz, S. A. S., \& Stumpf, I. R. C. (2014). Redes de colaboração na produção científica brasileira em biologia evolutiva: 2000-2012. In Iv anais do encontro brasileiro de bibliometria e cientometria (p. 1-7). Recife, Brasil.

Santin, D. M., Vanz, S. A. S., \& Stumpf, I. R. C. (2015). Produção científica em ciências biológicas da ufrgs: tendências temáticas no período 2000-2011. Perspectivas em Ciência da Informação, 20(3), 3-21. Recuperado de http://hdl.handle.net/20.500.11959/brapci/35103.

Scimago Journal Country Ranks . (2020). Scimago journal country ranks . Amsterdan: Elsevier. Recuperado de https:// www.scimagojr.com/.

Silva, \& Brisola, A. C. C. A. S. (2017). Panorama da produção científica dos docentes do programa de pós-graduação em biologia molecular e celular da unirio: 2011 a 2016. In Xviii encontro nacional de pesquisa em ciência da informação. Marília, Brasil.
Silva, Sobral, N. V., Santana, G. A., \& Cruz, T. L. (2012). Mapeamento da produção científica brasileira sobre acesso aberto: 2001 a 2011. Encontros Bibli: Revista Eletrônica de Biblioteconomia e Ciência da Informação, 17(esp 2), 19-35. doi: 10.5007/1518- 2924.2012v17nesp2p19.

Silveira, F. X., \& Teixeira, M. R. F. (2017). O conhecimento compartilhado em rede: um estudo da área de ciências biológicas e ciências ambientais do ifrs campus porto alegre (rs). In Xviii encontro nacional de pesquisa em ciência da informação. Marília, Brasil.

The world university rankings 2020. (2020). Times higher education. Recuperado de https://www .timeshighereducation.com/world-university-rankings.
Como citar este artigo (APA):

Freitas, J. L., Rosas, F. S. \& Mendes, S. L. (2020). A produção periódica científica afiliada ao Instituto Nacional da Mata Atlântica (INMA) na base Scopus (2009-2018). AtoZ: novas práticas em informação e conhecimento, 9(2), 32 - 43. Recuperado de: http:// dx.doi.org/10.5380/atoz.v9i2.75302 